\title{
X-ray polarization of solar flares measured with Rhessi
}

\section{Journal Article}

\section{Author(s):}

Suarez-Garcia, E.; Hajdas, W.; Wigger, C.; Arzner, K.; Güdel, M.; Zehnder, A.; Grigis, P.C.

Publication date:

2006-12

Permanent link:

https://doi.org/10.3929/ethz-b-000158085

Rights / license:

In Copyright - Non-Commercial Use Permitted

Originally published in:

Solar Physics 239(1-2), https://doi.org/10.1007/s11207-006-0268-1 


\title{
X-RAY POLARIZATION OF SOLAR FLARES MEASURED WITH RHESSI
}

\author{
E. SUAREZ-GARCIA, W. HAJDAS, C. WIGGER, K. ARZNER, \\ M. GÜDEL and A. ZEHNDER \\ Labor für Astrophysik, Paul Scherrer Institut, Villigen PSI, Switzerland \\ (e-mail: estela.suarez@psi.ch) \\ and \\ P. GRIGIS \\ Institute of Astronomy, Eidgenössische Technische Hochschule, Zurich, Switzerland
}

(Received 14 July 2006; accepted 26 September 2006; Published online 10 November 2006)

\begin{abstract}
The degree of linear polarization in solar flares has not yet been precisely determined despite multiple attempts to measure it with different missions. The high energy range, in particular, has very rarely been explored, due to its greater instrumental difficulties. We approached the subject using the Reuven Ramaty High Energy Spectroscopic Imager (RHESSI) satellite to study six X-class and $1 \mathrm{M}$-class flares in the energy range between 100 and $350 \mathrm{keV}$. Using RHESSI as a polarimeter requires the application of strict cuts to the event list in order to extract those photons that are Compton scattered between two detectors. Our measurements show polarization values between 2 and $54 \%$, with errors ranging from 10 to $26 \%$ in $1 \sigma$ level. In view of the large uncertainties in both the magnitude and direction of the polarization vector, the results can only reject source models with extreme properties.
\end{abstract}

\section{Introduction}

Measurements of the linear X-ray polarization in solar flares can provide essential information needed to identify the processes responsible for the acceleration of particles and the emission of radiation. For photon energies in the hard X-ray region, polarization is produced either through electron Bremsstrahlung or Compton scattering. The degree of polarization is usually a complex function of the strength and topology of the magnetic field. Thermal distributions of electron velocities result in small polarization values produced mainly by the conduction-driven anisotropy of the electrons in the primary source (Emslie and Brown, 1980) with some contribution from photons backscattered in the photosphere. Higher polarization levels are expected from nonthermal anisotropic distributions of electrons that are accelerated in well-ordered magnetic fields. In addition, for such cases, the observed polarization degree is related to the photon directivity (i.e., the anisotropy of the emitted radiation), which depends both on the electron beaming details and on the viewing angle.

Starting in the late seventies, several nonthermal models of X-ray emission from solar flares were developed (see McConnell et al., 2002 and references therein). 
Generally, the authors assume a uniform magnetic field perpendicular to the solar surface and electrons being accelerated toward the chromosphere (Elwert and Haug, 1970; Brown, 1972; Haug, 1972; Bai and Ramaty, 1978; Zharkova, Brown, and Syniavskii, 1995). These electrons, spiraling downwards along the magnetic field lines, produce Bremsstrahlung radiation in collisions with hot plasma. The polarization in the emitted X-rays is a function of the energy spectra of the electrons, their pitch angles (angle between their velocity vector and the magnetic field), and the column density distribution of the ambient plasma. Studying the spectral characteristics of the detected X-rays provides information about the electron energies, while the polarization is a very sensitive tool to sample the electrons' pitch angle distribution. Both for very small pitch angles, corresponding to high electron beaming, and for very large ones, the polarization degree can be equally high. The two cases can be distinguished by their polarization direction: parallel and perpendicular to the magnetic field line, respectively. The predicted polarization values can reach up to $60 \%$ at energies above $50 \mathrm{keV}$, being even higher at low energies (Haug, 1972). After introducing more realistic pitch angle distributions and taking into account photon backscattering processes in the photosphere, the expected polarization is reduced down to 20 or $30 \%$ (Brown, 1972; Bai and Ramaty, 1978).

Several more complex nonthermal models have been developed as well. In one of them, the magnetic field structure was defined as a semicircular loop anchored in the chromosphere (Leach and Petrosian, 1983). This approach allowed studying the $\mathrm{X}$-ray emissions separately from different parts of the loop. The highest polarization could be produced at the top (up to $85 \%$ ), while the photons observed from the footpoints (in the region of the dense chromosphere) would be polarized to the level of around $20 \%$.

In general, lower energies are predicted to yield stronger polarization signals (Haug, 1972; Bai and Ramaty, 1978; Leach and Petrosian, 1983), although the strength of this relation can vary depending on the model. In some recent theories, an opposite trend has also been reported (Zharkova, Brown, and Syniavskii, 1995). Experimental verification is usually difficult because the lowenergy part of the spectrum is strongly contaminated by a nonpolarized thermal emission.

In all the cases, the observed value of flare polarization is strongly dependent on the viewing angle. The highest polarization values are expected for large angles of view, when the line of sight is perpendicular to the magnetic field line. Thus, most theories predict higher polarization for flares located near the solar limb. Similar behavior is also expected for the directivity of the flare emission: the intensity of the emitted radiation should depend on the angle of view ( $\mathrm{Li}$ et al., 1994; Li, 1995). Constraints related to the model assumptions favor two possible directions of the polarization vector: either parallel to the plane defined by the magnetic field lines and the line of sight or perpendicular to it (Bai and Ramaty, 1978; Leach and Petrosian, 1983; Zharkova, Brown, and Syniavskii, 1995). 
Contrary to the intense theoretical work, only a few polarization measurements have been conducted in hard X-rays. In this energy range, the commonly used technique is based on Compton scattering (Lei, Dean, and Hills, 1997).

First attempts were done at energies around $15 \mathrm{keV}$ by Tindo et al. (1970, 1972) and Tindo, Shuryghin, and Steffen (1976) using polarimeter instruments on board of several Intercosmos satellites. Although initial results (Tindo et al., 1970) showed a linear polarization of around $40 \pm 20 \%$, the later studies of different flares (Tindo et al., 1972; Tindo, Shuryghin, and Steffen, 1976) found their polarization degrees always compatible with zero. Moreover, the data suffered from limited photon statistics and systematic errors related with the detector calibration.

In measurements of solar flare polarization with the Reuven Ramaty High Energy Spectroscopic Imager (RHESSI), Compton scattering can occur in a specially installed beryllium scatterer. This method can only be used at low energies (20$100 \mathrm{keV}$ ). Its details, together with RHESSI's polarimetric features, are described in McConnell et al. $(2002,2004)$.

The latest measurements at energies below $100 \mathrm{keV}$ have been performed with the SPR-N instrument on board of the Coronas-F satellite by Zhitnik et al. (2006). From a sample of 25 solar flares, these authors determined upper limits on the polarization degree in the range from 8 to $40 \%(3 \sigma)$. Only for the single case of the October 29, 2003 flare, they found a significant polarization degree, which increases from about $50 \%$ at energies $20-40 \mathrm{keV}$, up to more than $70 \%$ for the energy channel $60-100 \mathrm{keV}$.

Recently published, the only two measurements of solar flare polarization at high energies $(0.2-1 \mathrm{MeV})$, show $1 \sigma$ values of $21 \pm 10 \%$ and $-11 \pm 5 \%$ for one flare close to the limb and another near the Sun center, respectively (Boggs, Coburn, and Kalemci, 2006). In their studies, the authors applied the polarimetric capabilities of the RHESSI satellite in the coincidence mode, i.e., without using the Be-scatterer.

In this paper, we present results in the energy range from 100 to $350 \mathrm{keV}$, obtained for seven solar flares (X and $\mathrm{M}$ classes) also selected from the RHESSI instrument database. We used a method based on the scattering of photons from detector to detector of RHESSI that has previously been applied for polarization studies of gamma ray bursts (Coburn and Boggs, 2003; Rutledge and Fox, 2004; Wigger et al., 2004).

In Section 2, we explain how RHESSI can be used as a Compton polarimeter to study linear X-ray polarization at energies $\geq 100 \mathrm{keV}$. The flares selected for analysis, and the criteria used for their selection is detailed in Section 3. Monte Carlo simulations were performed to calculate the response of the instrument, and their results are discussed in Section 4. The final polarization results are described and compared with previous measurements in Section 5. The interpretation of the results is done in Section 6 by comparing with theoretical predictions. Finally, a brief summary of the conclusions of our work is given in Section 7. 


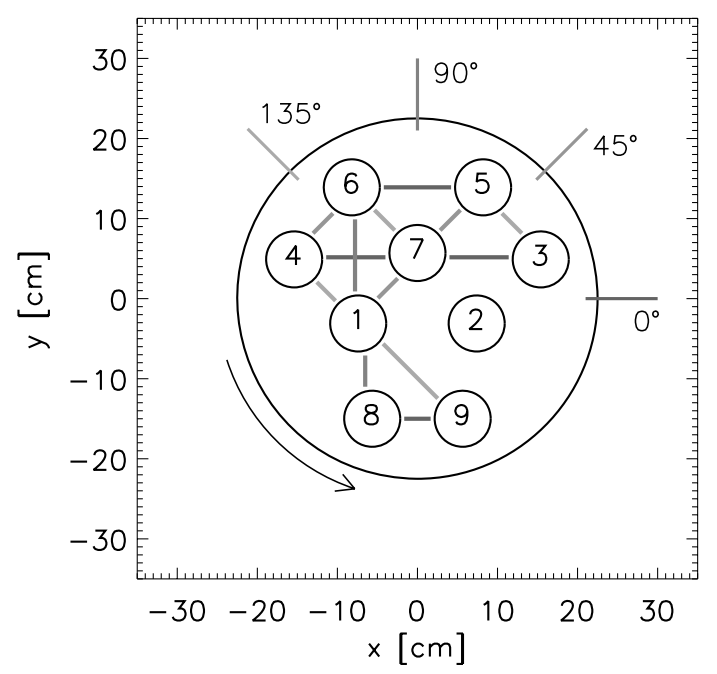

Figure 1. RHESSI detectors seen from the Sun. The grey lines indicate the possible scattering directions in the RHESSI fixed coordinate system (Section 2.4).

\section{Method Description}

\subsection{INSTRUMENT}

The Reuven Ramaty High Energy Spectroscopic Imager (Lin et al., 2002) was designed to observe solar flares from $3 \mathrm{keV}$ up to $17 \mathrm{MeV}$. It is capable of making spatially, spectrally, and temporally resolved images of the Sun (Hurford et al., 2002) using the rotation modulation principle (Schnopper, Thompson, and Watt, 1968; Skinner and Ponman, 1995). The spacecraft is rotating with a period $T \approx 4 \mathrm{~s}$. The RHESSI angular position in the solar coordinate system is calculated using the satellite roll angle, which is continuously monitored by the spacecraft aspect systems (Fivian et al., 2002; Hurford and Curtis, 2002).

The RHESSI spectrometer (Smith et al., 2002) consists of nine cooled Germanium detectors that are split in a thin front and a thick rear segment. Low-energy photons are mostly stopped in the front segments while high-energy photons can pass through and reach the rear segments. The energy resolution is in the order of a few $\mathrm{keV}$. The arrangement of the nine detectors in the spectrometer is sketched in Figure 1.

Each photon recorded by RHESSI is characterized by its arrival time, deposited energy, detector number, and segment. These parameters are stored in the RHESSI event list. The time resolution of RHESSI is equal to one binary microsecond (1b $\mu \mathrm{s}=2^{-20}$ seconds).

Polarization measurements are possible using photons that are Comptonscattered from one detector into another one, making a signal in both of them. 
TABLE I

Selection of cuts introduced to extract Compton scattered photons from the event list.

\begin{tabular}{ll}
\hline Criteria & Values \\
\hline Time interval & Between 1 and 4 minutes around the flare peak \\
Coincidence width & One binary microsecond \\
Single event energy & $25 \mathrm{keV}<E_{i}<300 \mathrm{keV}$ \\
Coincidence energy (sum) & $100 \mathrm{keV}<E_{i}+E_{j}<350 \mathrm{keV}$ \\
Kinematical cut & $\theta=90^{\circ} \pm 45^{\circ}$ \\
Detector segments & Rear segments (without detector 2) \\
Pairs selection & Neighbor detectors \\
Multiplicity & Two-event coincidences only \\
\hline
\end{tabular}

For such events, the effective area is very small, as most of the photons are either completely absorbed (in photopeak reactions) or scattered off the spectrometer (Hajdas, Wigger, and Zehnder, 2005). Therefore, only a small percentage of all photons observed by RHESSI produces more than one entry in the event list. The fact that RHESSI rotates allows reducing the systematic errors in the polarization analysis.

\subsection{COMPTON COINCIDENCES}

The Compton scattering probability of photons on free electrons is given by the cross section (Klein and Nishina, 1929):

$$
\frac{\mathrm{d} \sigma}{\mathrm{d} \Omega}=\frac{r_{0}^{2}}{2} \frac{E^{\prime 2}}{E^{2}}\left(\frac{E}{E^{\prime}}+\frac{E^{\prime}}{E}-2 \sin ^{2}(\theta) \cos ^{2}(\eta)\right)
$$

where $r_{0}$ is the classical electron radius, $\theta$ is the angle between photon infall and outfall direction, and $\eta$ is the angle between the infall polarization and the outfall direction. $E$ and $E^{\prime}$ are the energies of the photon before and after the scattering, respectively. The cross section $(\sigma)$ is minimal if $\eta=0^{\circ}$ and maximal if $\eta=90^{\circ}$. The dependence of the cross section on $\eta$ is most pronounced if $\theta=90^{\circ}$. As the RHESSI axis is pointing to the Sun, the detectors' plane is perpendicular to the photon infall direction, and therefore $\theta \approx 90^{\circ}$ for detector-to-detector scattering.

In order to extract Compton scattered photons from the RHESSI event list, several cuts were applied (see Table I).

As most of the high energy photons pass through the front segments without any interaction, only the rear segments were used for the polarization analysis. This condition ensures that Compton scattering events happen within the same binary microsecond (Wigger et al., 2004, Equation (8)). Detector 2 was not taken into account as it operates in a different way than the others (Smith et al., 2002). 
When more than two events happen at the same time, information about the polarization direction is lost. Therefore, we selected photons which were registered in exactly two detectors, calling them "coincidences." Furthermore, only coincidences between neighboring detectors were chosen, due to the very low probability of direct scattering between remote detectors (see Figure 1).

The sum of the two energies of the coincidence $\left(E_{i}\right.$ and $\left.E_{j}\right)$ had to fall into our selected range between 100 and $350 \mathrm{keV}$. In addition, the energy cut imposed on the individual detectors was in the range $25-300 \mathrm{keV}$. Below this region, the detector noise and the level of accidental coincidences (see Section 2.3) increased very strongly. A final constraint was applied by introducing the so-called kinematical cut that excludes coincidences that are incompatible with Compton scattering, using the kinematical relation between the scattering angle $\theta$ and the observed energies. The kinematical cut was especially useful to reject photons scattered from the Earth atmosphere (see Section 4).

Applying the above cuts, we obtain a raw list of coincidences that is still contaminated by background events of different origin.

\subsection{BACKGROUND SUBTRACTION}

Two major sources of background were taken into account in the following analysis. The first are accidental coincidences that occur when two independent solar photons are simultaneously detected. The second kind of background is not related to the flare itself but produced by cosmic rays and the cosmic gamma ray background.

The rate of accidental coincidences is proportional to the square of the incoming photon flux. In order to obtain the number of accidental coincidences $\left(N_{\text {acc }}\right)$ we repeated the same procedure that was used to find the raw list of coincidences (Table I), but taking pairs of events that are time delayed by 20 binary microseconds $<\mathrm{d} t<30$ binary microseconds (see Wigger et al., 2004). As the typical detector dead time is in the order of several microseconds, the delay length was chosen to be longer than this. The rate of accidental coincidences over this range is approximately constant and was used for background subtraction.

The second kind of background was determined either before or after the flare. For this purpose we selected time intervals shifted by 18 orbits, i.e., $24 \mathrm{~h}$, before or after the flare peak. In this way, the spacecraft geomagnetic coordinates were similar (see first part of Table II for an example), and the systematic effects coming from the background variations along the orbit were strongly reduced. Proper subtraction of this background requires some additional conditions: no other flare or high energy event should be present in that period, and the spacecraft operational status regarding attenuator state and decimation logic should be close to the one in the moment of the flare observation. The total number of background coincidences obtained in this way is denoted by $N_{\mathrm{bg}, \text { tot }}$. The period selected for background subtraction contains a (usually negligible) number $N_{\mathrm{bg} \text {,acc }}$ of accidental coincidences. It has to 


\section{TABLE II}

Geomagnetic coordinates of RHESSI satellite and numbers of coincidences determined at the peak of the flare on January 20, 2005 and during the background measurement period.

\begin{tabular}{lll}
\hline $\begin{array}{l}\text { Starting } \\
\text { time }\end{array}$ & $\begin{array}{l}\text { Peak of flare } \\
(06: 43: 00 \text { Jan 20, 2005) }\end{array}$ & $\begin{array}{l}\text { Background } \\
(06: 42: 59 \text { Jan 19, 2005) }\end{array}$ \\
\hline $\operatorname{RA}\left(^{\circ}\right)$ & -76.07 & -80.26 \\
Dec $\left(^{\circ}\right)$ & 37.91 & 37.91 \\
Total coincidences & $43313 \pm 208$ & $5874 \pm 77$ \\
Accidental coincidences & $26907 \pm 35$ & $66 \pm 2$ \\
Solar flare compton scattering coincidences & $10598 \pm 225$ & \\
\hline
\end{tabular}

Note. The data collecting time was 240 seconds.

be subtracted from $N_{\text {bg,tot }}$ because it is already included in $N_{\text {acc }}$ calculated during the flare peak. The number of coincidences produced by the nonflare related background is then $N_{\mathrm{bg}}=N_{\mathrm{bg}, \text { tot }}-N_{\mathrm{bg}, \text { acc }}$. As an example, the time evolution of the background signal observed one day before the January 20, 2005 flare is displayed together with the flare in Figure 2. It reproduces very well the background levels observed before and after the flare peak.

Finally, the rate of Compton scattering events was calculated for any time $t$ according to

$$
N_{C}(t)=N_{\mathrm{tot}}(t)-N_{\mathrm{acc}}(t)-N_{\mathrm{bg}}(t+\Gamma),
$$

where $\Gamma$ is chosen such that both the geomagnetic coordinates and the orientation (roll angle) of RHESSI are in the background period as close as possible to the

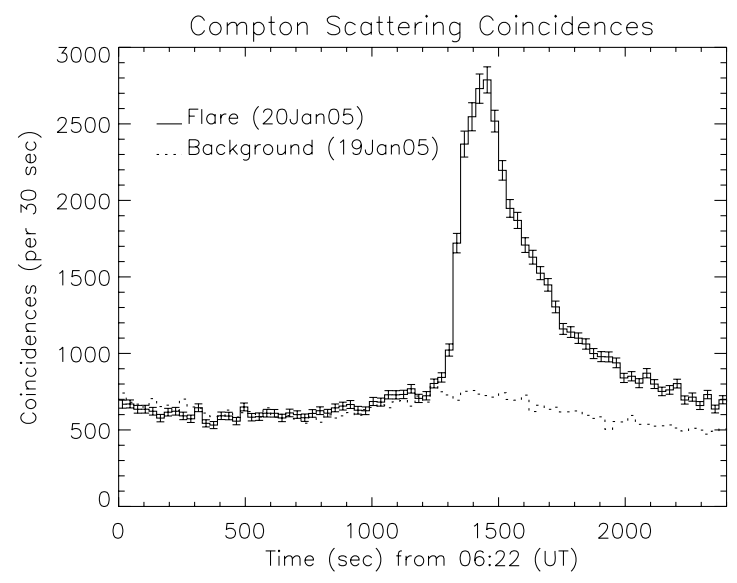

Figure 2. Signal and background coincidences versus time for the flare on January 20, 2005 together with the background levels measured approximately one day before at the same position. 
ones during the flare peak. $N_{\text {tot }}$ is the number of coincidences in the flare peak according to Table I, $N_{\mathrm{bg}}$ is the corresponding number of background events from flare-unrelated photons, and $N_{\text {acc }}$ is the number of accidental coincidences.

\subsection{POLARIZATION ANALYSIS}

For each coincidence, the line connecting the two detectors involved in the Compton scattering process defines the angle used to construct the modulation curve. According to the RHESSI fixed coordinate system, there are four possible scattering directions $d$ (labeled $0^{\circ}, 45^{\circ}, 90^{\circ}$, and $135^{\circ}$ ) between neighboring detectors (Figure 1). In each direction, the number $N_{d}\left(\tau_{i}\right)$ of coincidences per time bin $\tau_{i}$ (with $\left.\tau_{i}=t_{i} \operatorname{MOD} T\right)$ was divided by their sum in the same direction $\left(n_{d}\left(\tau_{i}\right)=\frac{N_{d}\left(\tau_{i}\right)}{\sum_{i} N_{d}\left(\tau_{i}\right)}\right)$. Such normalization is needed in order to eliminate the dependence on the number of detector pairs, which is different for each direction (see Figure 1).

The RHESSI roll period $T$ was measured using the spacecraft roll aspect system (Fivian et al., 2002; Hurford and Curtis, 2002) that provides the RHESSI angular position with an accuracy of one arcminute. We obtained the satellite rotation period for each flare using a linear fit. In order to verify its stability and check for possible drifts, $T$ was calculated in one second steps over a 720 seconds interval containing the flare. The maximum variations between the measurements were below one millisecond, which is negligible compared with the bin size used in the polarization analysis $(\approx 167$ milliseconds). It was also found that the drift of the period is smaller than $(2.1 \pm 2.0) \times 10^{-7}$. It corresponds to a change in the rotation period by less than 0.05 millisecond, giving an upper limit of $0.53^{\circ}(3 \sigma)$ on the possible phase shift for the longest time interval used in the analysis.

The asymmetry curve can be constructed as follows:

$$
A_{0-90}\left(\tau_{i}\right)=\frac{n_{0}\left(\tau_{i}\right)-n_{90}\left(\tau_{i}\right)}{n_{0}\left(\tau_{i}\right)+n_{90}\left(\tau_{i}\right)}
$$

The coincidences that occurred in the directions $45^{\circ}$ and $135^{\circ}$ were properly shifted and included in the equation stated earlier. Using asymmetry to determine the polarization, minimizes the effect of the lightcurve variations and grouping to have only two directions improves the statistics.

In order to relate the time variable $\tau$ with angular directions in the Sun, we used the relation: $\eta_{i}=-\frac{2 \pi}{T} \cdot \tau_{i}+\eta_{0}$. By convention, $\eta=0$ corresponds to solar West, and $\eta=\pi / 2$ corresponds to the solar North. $\eta_{0}$ is the angular position of RHESSI $\mathrm{X}$-axis $\left(0^{\circ}\right.$ direction from Figure 1$)$ with respect to the solar West in the moment when measurement started. Through this coordinate transformation, we obtained $A_{0-90}(\tau)$ in heliocentric coordinates: $A(\eta)$.

Due to the sinusoidal dependence of the Compton cross section for the scattering of a polarized photon (Equation (1)), the asymmetry curve is also a sinusoidal 
function with period equal to a half of the RHESSI rotation. It can be represented by a function:

$$
A(\eta)=\mu_{\mathrm{p}} \cdot \cos (2(\eta-\phi+\pi / 2)),
$$

where the amplitude $\mu_{\mathrm{p}}$ is a positively defined value equal to the flare modulation factor. The phase $\phi$ is the polarization angle from the flare.

Comparing the experimental amplitude $\mu_{\mathrm{p}}$ with the modulation factor $\mu_{100}$ from Monte Carlo simulations for a $100 \%$ polarized flux (Section 4), allows to determine the polarization degree of the solar flare (П) McConnell et al. (2002):

$$
\Pi=\frac{\mu_{\mathrm{p}}}{\mu_{100}} .
$$

Two parameters, $\Pi$ and $\phi$, fully describe the polarization state of a solar flare and are needed for comparison with theoretical predictions.

\section{Flares Selection}

The following criteria were applied to select the flares for polarization analysis: large intensity, strong high energy component, and negligible contamination with particles (either from the flare itself or from the radiation belts). Also, since theory predicts highest polarization close to the solar limb (Brown, 1972; Bai and Ramaty, 1978; Leach and Petrosian, 1983), we focused on limb-close flares. After applying all these conditions, we were able to select six $X$ and one M-class flares, five of them located within less than 120 arcs from the solar limb (Figure 3).

In order to study polarization in the most explosive part of the energy release, only the peak of the flare was chosen (see Figure 4). The time period for analysis varied between one and four minutes depending on the duration of the flare peak.

The spectrum of each flare was analyzed to find the energy ranges of different emission mechanisms. For this purpose, a fit was performed, using the RHESSI OSPEX fitting tool (Tolbert, 2006), with a combination of a thermal Bremsstrahlung curve and a broken power law (Figure 5). In all cases, the thermal contribution was found to be negligible at energies above $50 \mathrm{keV}$. For further analysis, we selected the nonthermal Bremsstrahlung region (100-350 keV). The spectral indices are given in Table III and correspond to a single power law fit of that part of the flare. These values were subsequently used in the simulations performed to determine the instrumental response function and its polarization modulation factor (see Section 4). Although photons with energies between 50 and $100 \mathrm{keV}$ are already in the nonthermal emission region, their interaction in the RHESSI detectors is governed by photoelectric absorption, leaving only a marginal number of Compton scattering events. On the high-energy side, the threshold value was chosen to avoid regions dominated by the background.

The main parameters that describe the flares selected are summarized in Table III. 


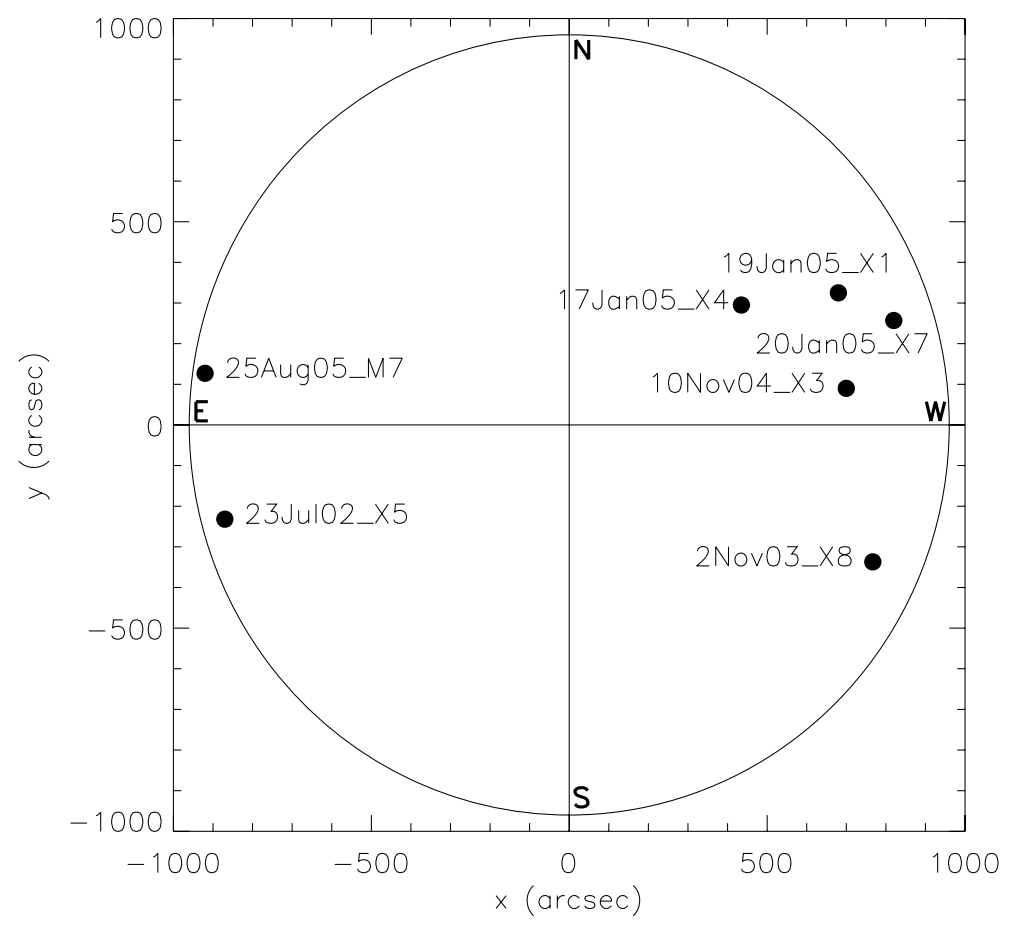

Figure 3. Distribution of the flares in the solar disc. Their position was extracted from images taken with RHESSI.

\section{Simulations}

Monte Carlo simulations have been performed to calculate the response of the RHESSI polarimeter to a $100 \%$ polarized solar flare. For this purpose, the exact mass model of the whole satellite and its germanium spectrometer has been constructed and implemented in the GEANT 3.21 simulation code (CERN, 1994). The spacecraft was illuminated by a uniform beam of photons coming parallel to the RHESSI rotation axis. Each incoming photon was fully tracked and all energy depositions made on its path in any of RHESSI detectors were recorded. In this way, a simulated event list was created and subsequently used to determine the modulation factors. The procedure to extract the modulation factors from the event list was the same as for the analysis of the solar flares (Section 2), but neglecting the accidental coincidences. We made two sets of simulations:

1. The modulation factors for $100 \%$ polarized emissions were determined for each flare using a photon energy distribution with spectral indices as given in Table III. The incoming photons were $100 \%$ polarized and their energy range was either $100-600$ or $80-350 \mathrm{keV}$. It was found that the contribution from photons above 


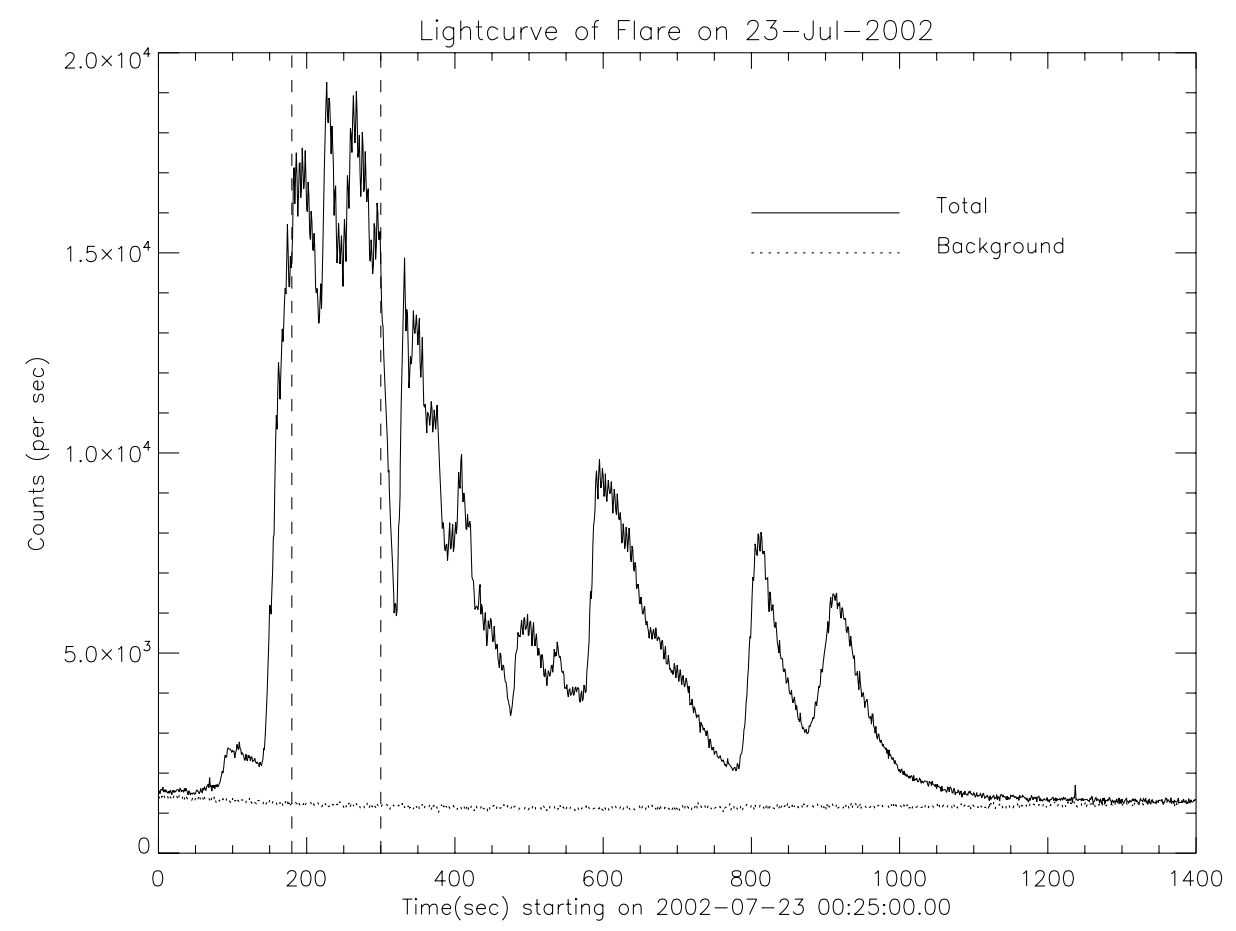

Figure 4. Ligthcurve of the solar flare on July 23, 2002 and background selected for it. The number of single events registered in rear segments of all detectors (except number 2), with energies between 25 and $300 \mathrm{keV}$ is plotted as a function of time. The part of the flare used for the polarization measurement is enclosed by the two vertical dashed lines.

$350 \mathrm{keV}$ to the modulation curve in our energy range $(100-350 \mathrm{keV})$ was negligible. An example of a modulation curve is shown in Figure 6.

2 . In the second case, we took monoenergetic photons with $100 \%$ polarization at 13 energies between 100 and $1000 \mathrm{keV}$ (Figure 7). The mean modulation factor of each flare was subsequently calculated by averaging the monoenergetic modulation factors. The weights used for the average were proportional to the number of coincidences per energy bin. $\mu_{100}$ is maximum around $170 \mathrm{keV}$. It decreases for lower energies due to the low energy threshold $(25 \mathrm{keV})$, which only allows detecting recoil electrons from photons scattered at very large angles. At such angles, modulation factor of the instrument is very small, in accordance with Equation (1). Above $170 \mathrm{keV}, \mu_{100}$ diminishes with energy following the polarization sensitivity based on Equation (1) for photons scattered around $90^{\circ}$. Using two exponential functions with properly chosen coefficients reproduces the above features in a simple way (see fit function in the caption of Figure 7).

Both approaches gave the same results. For example, for the case of the July 23, 2002 flare, with a spectral index equal to -3.1 , we obtained with the first method $\mu_{100}=32.8 \pm 1.6 \%$ and with the second one $\mu_{100}=32.4 \pm 5.4 \%$. 


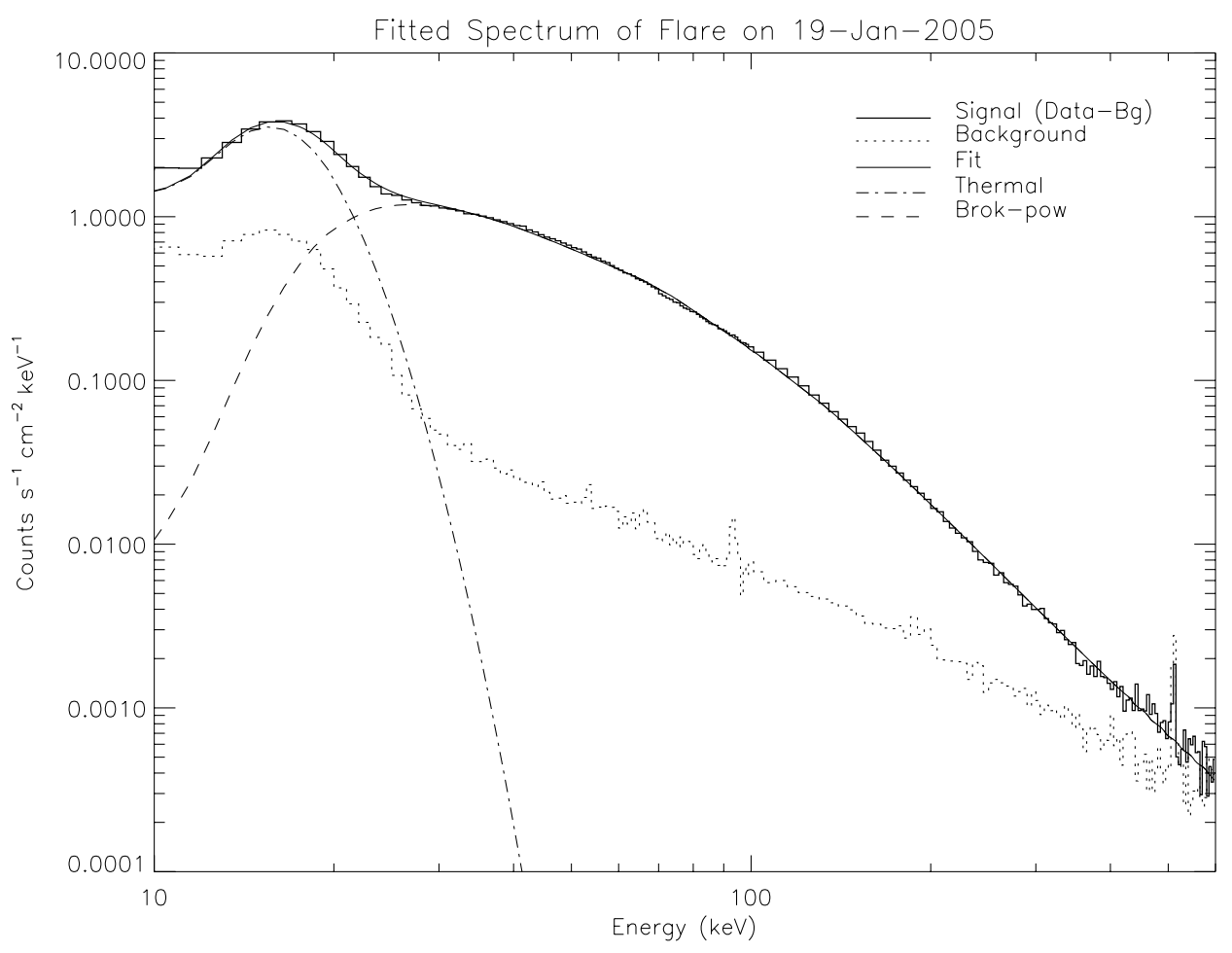

Figure 5. Background-subtracted spectrum of the flare on January 19, 2005. The fit was made for energies between 12 and $600 \mathrm{keV}$ combining a thermal curve and a broken power law. Thermal component is negligible above $40 \mathrm{keV}$, and the background becomes dominant around $500 \mathrm{keV}$.

The analysis of the solar flare lightcurves from single detectors revealed periodic structures that can be attributed to photons reaching RHESSI after being scattered by the Earth's atmosphere. The contamination of the modulation curves by such photons was computed with another set of Monte Carlo simulations. For this purpose, a simplified system consisting of the satellite and the Earth with its atmosphere was constructed. The Earth was represented by a solid sphere with 12 layers of atmosphere extending up to about $50 \mathrm{~km}$ above the surface. The mass as well as the chemical composition of all the atmospheric sheets were equal, while the density varied in accordance with their height.

Simulations of Earth scattering were performed using unpolarized photons with spectral indices typical for the analyzed flares and their corresponding angular positions between RHESSI, the Earth, and the Sun. The largest fraction of photons detected from the atmosphere was found when the Earth reached the angle of $90^{\circ}$ with respect to the RHESSI-Sun direction. In the energy range $100-350 \mathrm{keV}$, up to $30 \%$ of the observed photons were coming from the Earth, producing a strong modulation in the single-event lightcurves (Figure 8, solid line). The influence of such photons on the asymmetry curves, extracted from coincidences, was much 


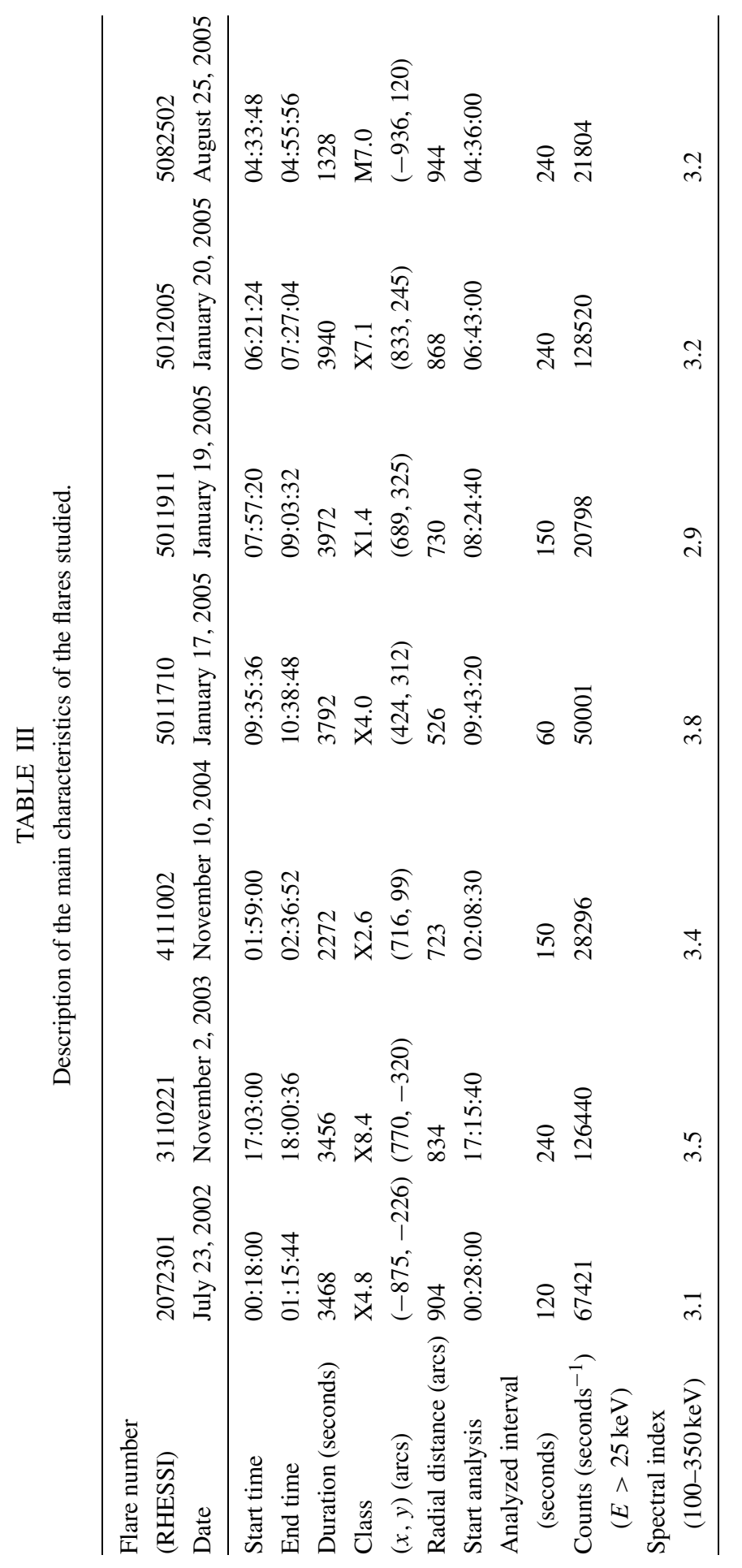




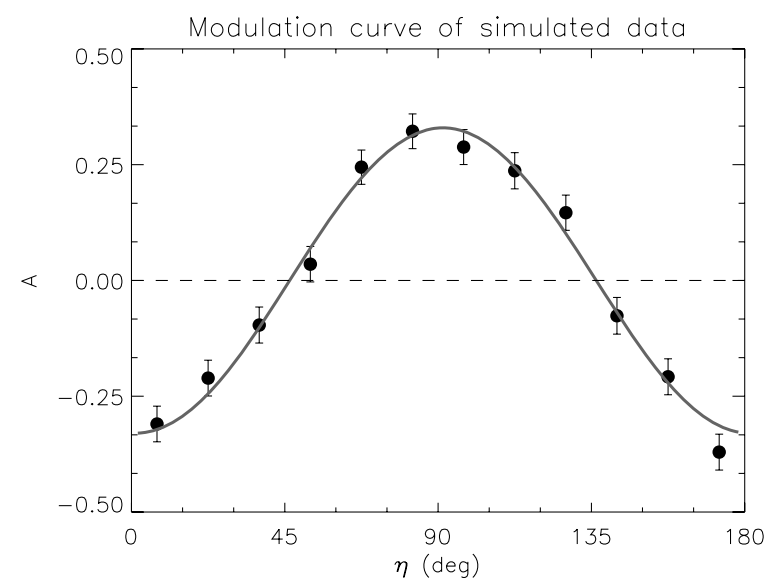

Figure 6. Simulated asymmetry plot for a $100 \%$ polarized flare with spectral index -3.1 .

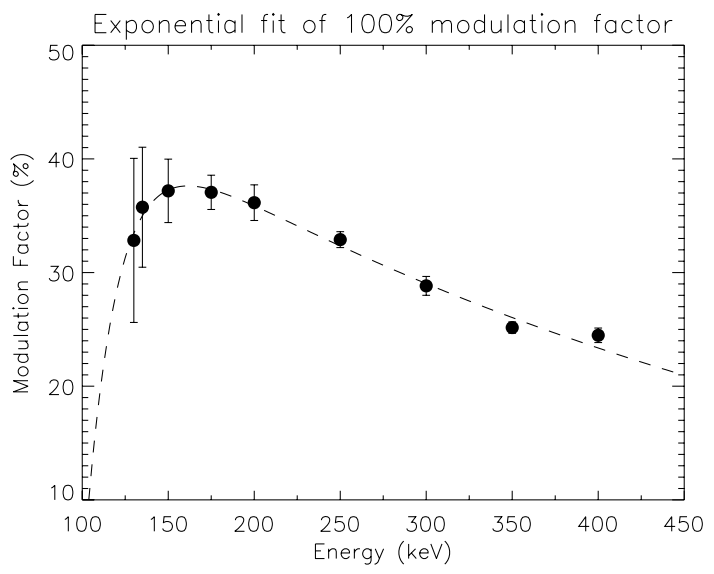

Figure 7. Simulated energy dependence of $\mu_{100}$. The dashed line corresponds to the fit function: $\mu_{100}=56 \cdot e^{-E / 460}-8668 \cdot e^{-E / 19}$.

smaller. Most of the Earth scattering caused only accidental coincidences or did not pass the kinematical cut. Finally, the contamination by the Earth-scattered photons was, in the worst case, less than $8 \%$. Considering their low level and flat distribution along the modulation curves, the modifications of the measured modulation factors were negligible compared to the overall statistical error.

The presence of the grids above the detectors could produce periodic structures also in the coincidence lightcurves and, therefore, this effect was carefully studied with Monte Carlo simulations. Those sources which are not in the center of the Sun are, during RHESSI rotation, intermittently obscured by the grids. This produces in their single-event lightcurves a modulated profile with $180^{\circ}$ periodicity (Hurford et al., 2002) and such a pattern could be mixed up with the real polarization signal. First, the lightcurve modulation is strongly reduced for high energy photons 


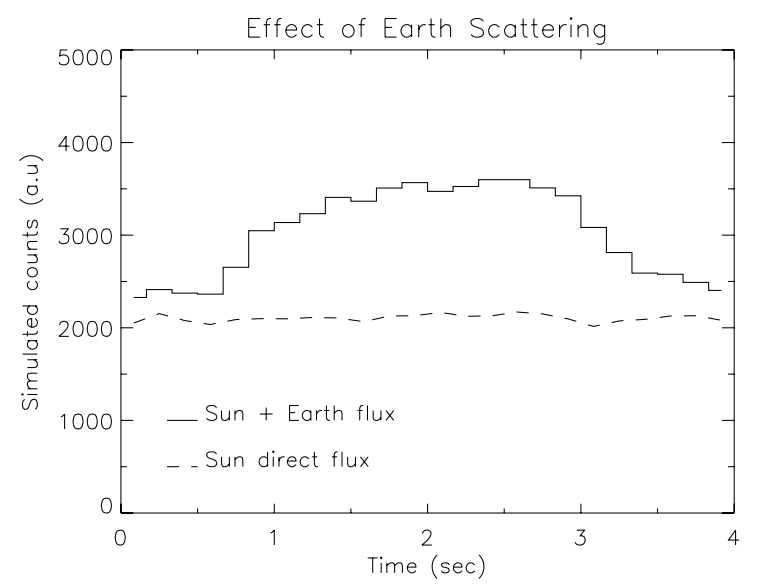

Figure 8. Simulations of scattering by the Earth's atmosphere. The figure shows single events with energies between 100 and $350 \mathrm{keV}$ arriving at the rear part of detector number 4 . The solid line shows the total photon flux, while the flux coming directly from the Sun appears as a dashed line.

reaching the rear detectors. Second, the width of the angular bin used for polarization analysis is large enough to average all count-rate variations caused by the grid modulation. The lightcurve variations further cancel out since the grids of detector pairs used for coincidences are generally aligned in different directions.

We performed simulations of a flare situated in the solar limb where the grid effect would be the strongest. Fine grids have been approximated keeping the slit/slat width ratio while the coarse ones were exactly implemented. The modulation factor obtained for a nonpolarized flux of $10^{8}$ photons in the $100-350 \mathrm{keV}$ energy range and with a power law spectrum index of -3.1 , was $2.5 \pm 1.9 \%$. This is similar to the signal measured from a nonpolarized source situated in the center of the sun, where grids do not cause any modulation. Therefore, the grids' effect can be neglected.

Notice that due to the positive definiteness of the polarization degree, even an unpolarized signal gives a nonvanishing amplitude in the asymmetry plot when applying to our analysis. From a 0\% polarized simulated flare with around 8500 coincidences, we obtained a modulation factor equal to $(3.4 \pm 1.6) \%$.

\section{Results}

\subsection{RHESSI POLARIZATION MEASUREMENTS}

The asymmetry curves $A\left(\eta_{i}\right)$ are displayed in Figure 9, together with the best fit of the function in Equation (4). These curves have a periodicity of $T / 2$ ( $T$ equal to RHESSI rotation period). To improve the statistics, the second half of the asymmetry curves was added to their first half, plotting only the range $0-180^{\circ}$. 

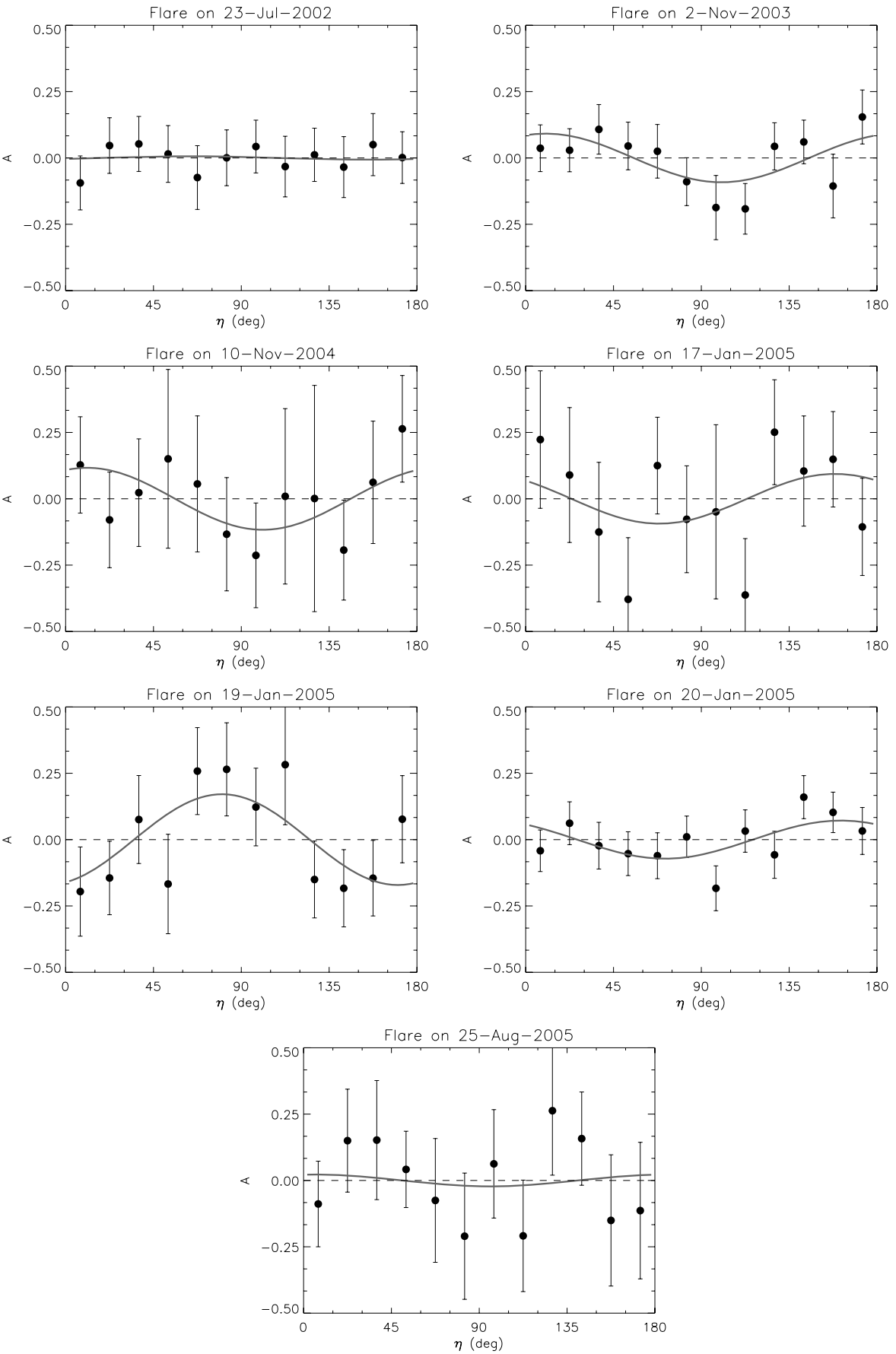

Figure 9. Asymmetry curves of all flares analyzed, extracted for photon energies between 100 and $350 \mathrm{keV}$. The thick lines show the best fits with the function from Equation (4). The angle of the minimum in the fit curve indicates the flare polarization direction in heliocentric coordinates. 
The degrees of polarization $\Pi$ were calculated using Equation (5) with $\mu_{100}$ taken from simulations. The mean value of $\mu_{100}$ was $33.2 \%$ and its variations between different flares were below $1.1 \%$. The resulting polarization degrees were found to be between 2 and 54\%, with error bars varying from 10 to $26 \%$ at the $1 \sigma$ level (see Table IV). The $\Pi$ values of all the flares are plotted versus the flare class in Figure 10, where no significant correlation between these two quantities can be observed.

The data does not show any preferential direction of polarization. When plotting the polarization angles of the flare sample in heliocentric coordinates (Figure 11, left), the points seem to concentrate around the North-South solar direction but the error bars are too large to extract a firm conclusion. When the angle between the polarization direction and the line that joins the flare position and the center of the Sun was calculated, no tendency was found (Figure 11, right).

Despite the very high single-photon count rates of more than 20000 counts per second, the mean number of Compton events found per flare was only around 4000 , and the smallest one does not even reach 1000 coincidences. This reflects the small value of the RHESSI effective area for Compton polarimetry as discussed in Section 2. The signal-to-background ratio is on the average only around 0.5. Depending on the flare, the largest background contribution is produced either by accidental coincidences or by cosmic $\gamma$-ray background. The number of counts found with each flare are compiled in the first part of Table IV, where the errors refer to $1 \sigma$ and are purely statistical.

\subsection{COMPARISON WITH OTHER MEASUREMENTS}

With respect to the polarization amplitude, our results are consistent with previous measurements made at higher energies by Boggs, Coburn, and Kalemci (2006). In the particular case of the July 23, 2002 flare that they also analyzed, our value $(2 \pm 14 \%)$ is smaller than theirs $(21 \pm 10 \%)$, but agreement is found at the $1.5 \sigma$ level. The difference can be explained by the different time periods and energy ranges used in both cases. Extending the time and energy windows for our analysis toward the values selected by Boggs, Coburn, and Kalemci (2006) provides very similar polarization levels. The polarization angles of the two flares measured by Boggs, Coburn, and Kalemci (2006) are aligned along the North-South direction in heliocentric coordinate system. However, these authors conclude that polarization is azimuthal for near-limb flares, but radial for those close to the Sun center. We cannot confirm this rule from our observations (see Figure 11, right). The values found in the present work are more uniformly distributed between 35 and $85^{\circ}$, independently of the flare location. Taking into account the values of the error bars and the size of the statistical sample of analyzed flares, further measurements aimed to verify the observed disparity are needed. 


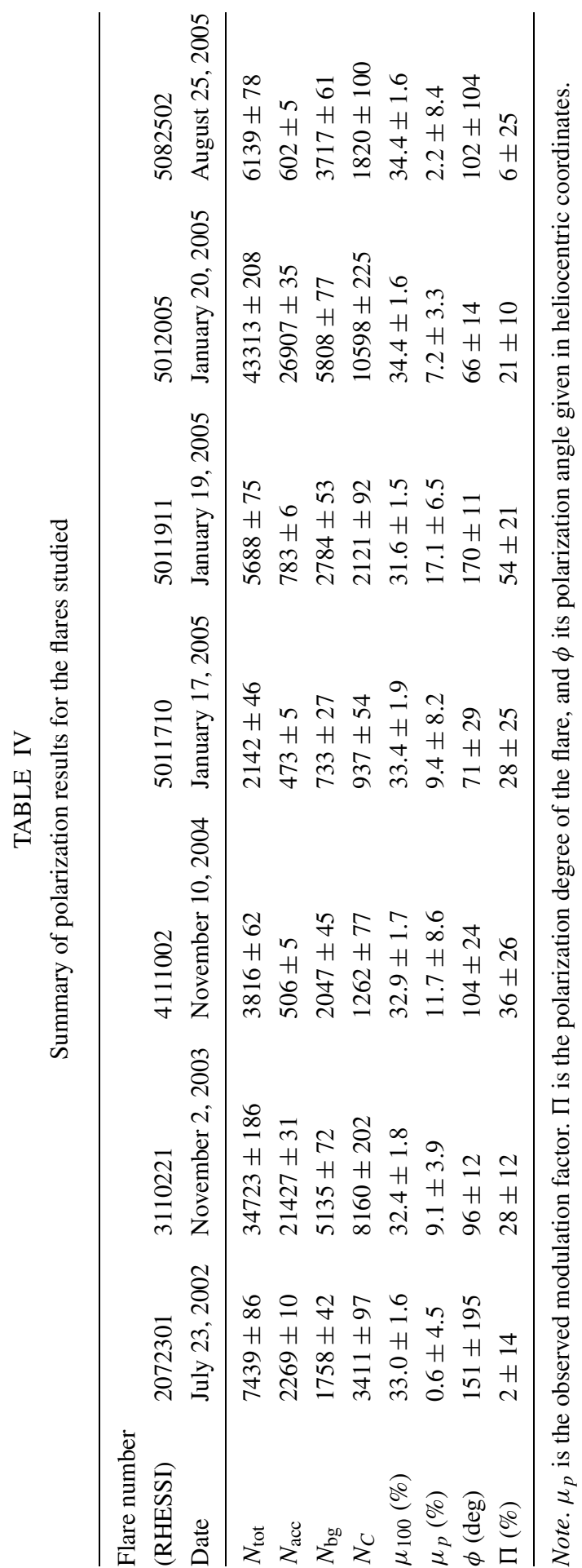




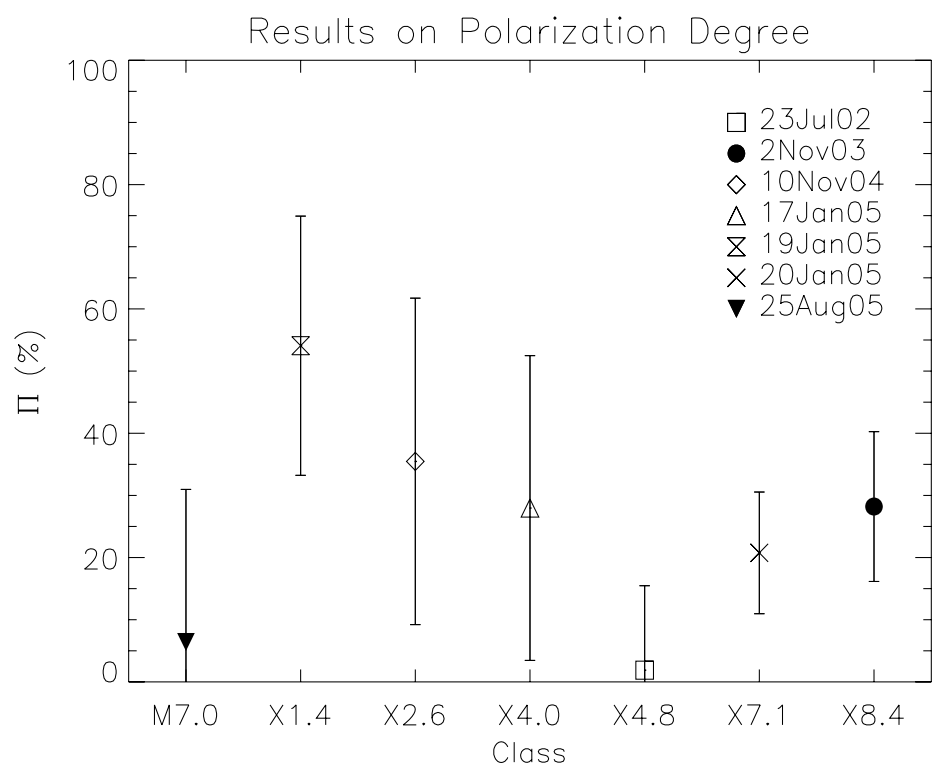

Figure 10. Results on the degree of polarization, with their $1 \sigma$ error bars.
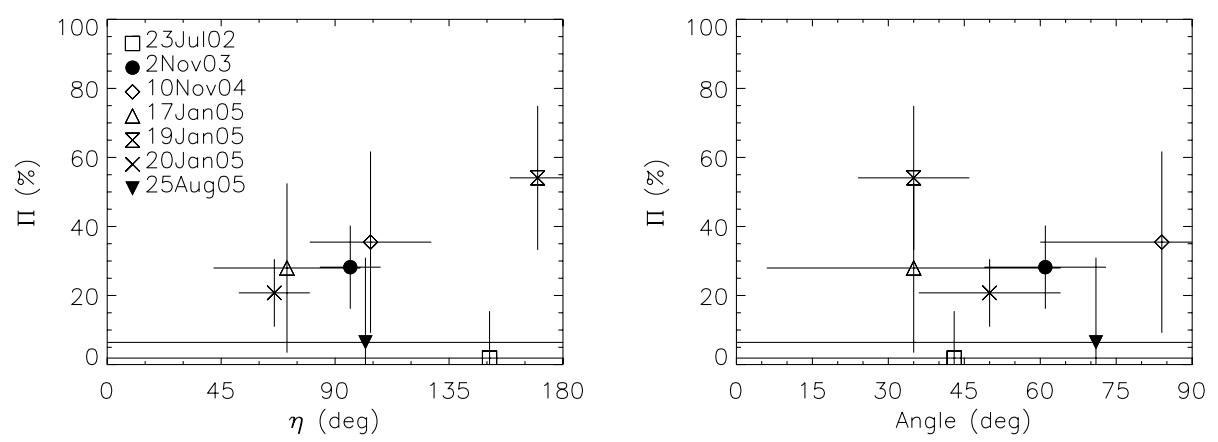

Figure 11. Polarization degree versus polarization angle plotted in two different reference systems: with respect to solar equator (left) and with respect to the radial line that joins the center of the Sun and the flare position (right).

Recent polarization data at energies up to $100 \mathrm{keV}$ have became available from measurements with the SPR-N instrument on board of the Coronas-F satellite (Zhitnik et al., 2006). From a sample of 25 solar flares, upper limits on the polarization degree were found to be in the range of $8-40 \%(3 \sigma)$. These values are in good agreement with our results (typically within $2 \sigma$ level). In particular, for the flare on January 20, 2005, observed simultaneously by both satellites, the polarization value from RHESSI observations was equal to $21 \pm 10 \%$ while the upper limit in the Coronas-F measurement was equal to $17 \%$. Again, a more direct comparison is not possible because both the energy range and the time intervals analyzed were 
different. For one flare (on October 29, 2003), the Coronas-F instrument showed a significant polarization degree that increases from about $50 \%$ at energies 20 $40 \mathrm{keV}$, up to more than $70 \%$ for the energy channel $60-100 \mathrm{keV}$. Unfortunately, RHESSI polarization analysis of the October 29, 2003 flare was not possible due to a high contamination of its detectors with charged particles.

\section{Interpretation}

Our results were compared with theoretical predictions for the nonthermal photon emission given by Bai and Ramaty (1978), Leach and Petrosian (1983) and also with the $0 \%$ polarization hypothesis. Bai and Ramaty (1978) provide the most comprehensive set of theoretical data, covering the whole energy range from 10 to $500 \mathrm{keV}$. These authors considered primary X-ray emission due to Bremsstrahlung of the accelerated electrons moving toward the photosphere, adding also the X-ray Compton backscattering component. The photon polarization was studied for different electron spectra and pitch angle distributions and results were presented as a function of the observing angle. At energies around $200 \mathrm{keV}$, typical for our analysis, the polarization reaches maximum values between 20 and $30 \%$ and decreases quickly for flares located closer to the solar center. Taking this trend into account, the predicted polarization degrees for our sample of flares range from -19 to $1 \%$, where the negative sign indicates a polarization direction parallel to the magnetic field, and the positive one perpendicular to it.

Leach and Petrosian (1983) analyzed emissions from more complex, loopshaped magnetic fields, but most of their polarization prediction is given only for two energies: 16 and $102 \mathrm{keV}$. As the dependence of the polarization on the energy was weak, we extrapolated their values toward our energy range. The highest polarizations for large energies are expected to come either from the top of the flare loop, or from the transition region above the chromosphere. The latter option roughly corresponds to RHESSI observations in which the high-energy emissions come from the footpoints. Nevertheless, a clear distinction between chromospheric and transition zone emission was not possible. Leach and Petrosian (1983) proposed several models depending on the magnetic field gradient, the electron pitch angle distribution, and the spectral index. We selected three different cases for comparison purposes: one with a homogeneous magnetic field and a uniform distribution of the electron pitch angles (model 3 ), another with equally homogeneous magnetic field but pitch angles close to $90^{\circ}$ (model 5), and the last one with a large magnetic field gradient and large pitch angles (model 8). After correcting for the flare position on the Sun, the expected polarization degrees of our flares are in the ranges between $-7 \%$ and $18 \%$ for model 3 , from -45 to $-80 \%$ for model 5 , and from $-6 \%$ to $23 \%$ for model 8 .

The latest theoretical work on polarized emission, with the most advanced electron beam dynamics, was presented by Zharkova, Brown, and Syniavskii (1995). 
Polarization calculations were done only up to $100 \mathrm{keV}$ (for photon spectral indices typical to our flares), and showed increased values for higher energies. Their predictions, extrapolated to the energy range used in the present work, are similar to those from Bai and Ramaty (1978) and cannot be distinguished by the following $\chi^{2}$ analysis.

The comparison of our observations with the models was done by generating asymmetry curves for the flares polarized in accordance with the theoretical predictions. Monte Carlo simulations were performed for each flare individually, using exactly the same RHESSI mass model as in Section 4. The expected polarization angle and amplitude were properly determined by adjusting the theoretical predictions to the flare position on the Sun. The high number of simulated events allowed to keep the statistical errors on a very low level compared with the experimental data. Finally, the reduced $\chi^{2}$ value was calculated using the data points from all seven flares as given by Equation (6).

$$
\chi^{2}=\frac{1}{n(m-1)} \sum_{i=1}^{n} \sum_{j=1}^{m} \frac{\left(N_{i, j}^{e}-N_{i, j}^{t}\right)^{2}}{\left(\Delta N_{i, j}^{e}\right)^{2}+\left(\Delta N_{i, j}^{t}\right)^{2}},
$$

where $i$ is the flare number and $j$ is the angular bin in the asymmetry plot. $N_{i, j}^{e}$ and $N_{i, j}^{t}$ are the experimental and theoretical numbers of coincidences, respectively, and $\Delta N_{i, j}^{e}$ and $\Delta N_{i, j}^{t}$ are their statistical uncertainties. The total number of degrees of freedom was equal to 77 . The results for all four models and the $0 \%$ polarization hypothesis are displayed in Table V.

From the $\chi^{2}$ values of Table $\mathrm{V}$ we can reject the model 5 from Leach and Petrosian (1983), with a $90 \%$ of confidence. For the rest of the models, the $\chi^{2}$ are very close to unity, preventing us from distinguishing between them. Within the error bars, they all agree with the experimental data equally well. The same is also valid for the $0 \%$ polarization hypothesis. Further refinement would require a polarimeter that is able to deliver data with error bars on the level of $1-2 \%$.

\section{TABLE V}

Reduced $\chi^{2}$ from all the flares combined, obtained by comparison of several theories with our measurements.

\begin{tabular}{ll}
\hline Model & Reduced $\chi^{2}$ \\
\hline Bai and Ramaty (1978) & 1.03 \\
Leach and Petrosian (1983) (model 3) & 0.86 \\
Leach and Petrosian (1983) (model 5) & 2.70 \\
Leach and Petrosian (1983) (model 8) & 0.83 \\
0\% polarization & 0.82 \\
\hline
\end{tabular}




\section{Summary and Conclusions}

Measurements of hard X-ray polarization have been performed for six X-class and one M-class flares from the RHESSI database. Our flare sample was identified after applying selection criteria to the signal strength, background levels, and level of contamination by charged particles in RHESSI detectors. The selected energy range, from 100 to $350 \mathrm{keV}$, connects the old (Tindo et al., 1970, 1972; Tindo, Shuryghin, and Steffen, 1976) and new (Zhitnik et al., 2006) results at low energies with the only measurement reported at a high energy band (Boggs, Coburn, and Kalemci, 2006).

We found values for the polarization degree in the range between 2 and 54\%, with statistical errors from 10 to $26 \%$ at the $1 \sigma$ level. The polarization angles are distributed between $66^{\circ}$ and $170^{\circ}$ in heliocentric coordinates. They do not show any preferential orientation of the polarization, neither parallel nor perpendicular, with respect to the radial direction defined by the position of the flare in the Sun. In addition, no significant dependency between the orientation of the polarization and the distance of the flares to the Sun center was found.

The polarization orientation with respect to the line that joins the two major footpoints of the flare was also studied. For this purpose, images of the flares were constructed with RHESSI at different energies. The emission above $\sim 30 \mathrm{keV}$ was found to be produced around the footpoints. However, no correlation between polarization direction and footpoints' orientation was found. Similarly, no relationship between solar flare intensity and polarization degree could be observed.

The polarization degree from the July 23, 2002 flare measured by Boggs, Coburn, and Kalemci (2006) at high energies is in agreement with our results at the $1.5 \sigma$ level. Their conclusion about the orientation of polarization respect to the radial direction passing trough the flare position (perpendicular to it for flares in the limb and parallel for flares close to center) cannot be confirmed by our measurements. Our angles are distributed between 35 and $85^{\circ}$ independently of the flare location. Comparison with theoretical predictions is more complex, as the direction of the polarization is expected by the theory to change around $300 \mathrm{keV}$ (Bai and Ramaty, 1978). Again, more observations with better accuracy are needed.

Regarding the low energy measurements, our data are in good agreement with the recent results from the SPR-N instruments on board the Coronas-F satellite (Zhitnik et al., 2006), typically within $2 \sigma$. Their large sample of 25 solar flares reveals low polarization degrees, providing $3 \sigma$ upper limits from 8 to $40 \%$. Unfortunately, no information is given about the polarization direction. In the October 29, 2003 flare, for which the Coronas-F team claims a polarization of $70 \%$, RHESSI measurements were contaminated by charged particles.

Theoretical predictions of the nonthermal Bremsstrahlung emission provide polarization levels of the order of $20 \%$. However, depending on the assumptions used by different authors, the expected polarization can differ not only by its value, but also by its orientation (see, for example, the calculations at $100 \mathrm{keV}$ from Bai 
and Ramaty (1978) and Leach and Petrosian (1983)). As the statistical uncertainties provided by our instrument are in the same order as the model predictions, equally good agreement is found for any of them as well as for the case of $0 \%$ polarization. In order to distinguish between different models, polarimetry measurements of at least $2 \%$ accuracy are needed. Only model number 5 from Leach and Petrosian (1983), predicting polarization up to $85 \%$, could be rejected by our RHESSI data. In this model, the magnetic field has the same strength at the top and the bottom of the loop, and the electrons spiral at pitch angles close to $90^{\circ}$.

RHESSI has made the first steps toward the understanding of the polarization phenomena in solar flares above $100 \mathrm{keV}$, where nonthermal emission dominates. Measurements with accuracy better than 10-20\% were, however, hardly possible. This is due to its small effective area and high levels of flare-induced background. Continuation of such studies will require a dedicated polarimeter that must solve the problems inherent to the RHESSI design. Emphasis should be put on increasing the effective area and improving the background rejection capabilities. In particular, a better time resolution will reduce the number of accidental coincidences, and the optimization of the detector dimensions will improve the detection efficiency for the Compton scattering.

\section{References}

Bai, T. and Ramaty, R.: 1978, Astrophys. J. 219, 705.

Boggs, S.E., Coburn, W., and Kalemci, E.: 2006, Astrophys. J. 638, 1129.

Brown, J.C.: 1972, Solar Phys. 26, 441.

CERN 3.21, Program Library Long Writeup W5013, October 1994 edn.

Coburn, W. and Boggs, S.E.: 2003, Nature 423, 415.

Elwert, G. and Haug, E.: 1970, Solar Phys. 15, 234.

Emslie, G.A. and Brown, J.C.: 1980, Astrophys. J. 237, 1015.

Fivian, M., Henneck, R., Mchedlishvili, A., and Zehnder, A.: 2002, Solar Phys. 210, 87.

Hajdas, W., Wigger, C., and Zehnder, A.: 2005, Nuovo Cimento 28, 817.

Haug, E.: 1972, Solar Phys. 25, 425.

Hurford, G.J. and Curtis, D.W.: 2002, Solar Phys. 210, 101.

Hurford, G.J., Schmahl, E.J., Schwartz, R.A., Conway, A.J., Aschwanden, M.J., Csillaghy, A., et al.: 2002, Solar Phys. 210, 61.

Klein, O. and Nishina, Y.: 1929, Astrophys. J. 52, 853.

Leach, J. and Petrosian, V.: 1983, Astrophys. J. 269, 715.

Lei, F., Dean, A.J., and Hills, G.L.: 1997, Space Sci. Rev. 82, 309.

Li, P.: 1995, Astrophys. J. 443, 855.

Li, P., Hurley, K., Barat, C., Niel, M., Talon, R., and Kurt, V.: 1994, Astrophys. J. 426, 758.

Lin, R.P., Dennis, B.R., Hurford, G.J., Smith, D.M., Zehnder, A., Harvey, P.R., et al.: 2002, Solar Phys. 210, 3.

McConnell, M.L., Ryan, J.M., Smith, D.M., Lin, R.P., and Emslie, A.G.: 2002, Solar Phys. 210, 125.

McConnell, M.L., Smith, D.M., Emslie, A.G., Hurford, G.J., Lin, R.P., and Ryan, J.M.: 2004, COSPAR Proc. [edn., Elsevier, New York] 34, 462.

Rutledge, R.E. and Fox, D.B.: 2004, Mon. Not. R. Astron. Soc. 350, 1288. 
Schnopper, H.,W., Thompson, R.I., and Watt, S.: 1968, Space Sci. Rev. 8, 534.

Skinner, G.K. and Ponman, T.J.: 1995, Inverse Probl. 10, 655.

Smith, D.M., Lin, R.P., Turin, P., Curtis, D.W., Primbsch, J.H., Campbell, R.D., et al.: 2002, Solar Phys. 210, 33.

Tindo, I.P., Shuryghin, A.I., and Steffen, W.: 1976, Solar Phys. 46, 219.

Tindo, I.P., Ivanov, V.D., Mandelstam, S.L., and Shuryghin, A.I.: 1970, Solar Phys. 14, 204.

Tindo, I.P., Ivanov, V.D., Mandelstam, S.L., and Shuryghin, A.I.: 1972, Solar Phys. 24, 429.

Tolbert, K. 'OSPEX - Object Spectral Executive', 2006, http://hesperia.gsfc.nasa.gov/ssw/packages/ spex/doc/ospex_explanation.htm.

Wigger, C., Hajdas, W., Arzner, K., Güdel, M., and Zehnder, A.: 2004, Astrophys. J. 613, 1088.

Zharkova, V.V., Brown, J.C., and Syniavskii, D.V.: 1995, Astron. Astrophys. 304, 284.

Zhitnik, I.A., Logachev, Y.I., Bogomolov, A.V., Denisov, Y.I., Kavanosyan, S.S., Kuznetsov, S.N., et al:: 2006, Solar Syst. Res. 40, 93. 